\section{Sensitivity of electroen- cephalogram in the diagnosis of epilepsy among patients attending a neuropsychiatric hospital in Northern Nigeria}

\author{
Shehu Sale \\ Federal Neuropsychiatric Hospital, \\ Kware, Sokoto State, Nigeria
}

\begin{abstract}
Electroencephalography (EEG) is an essential adjunct in management of epilepsy. While some studies reported that EEG has a high sensitivity in detecting epileptic seizures, others disagree. Therefore, it is important to know sensitivity of this important procedure in our setting. This was a cross-sectional study carried out from 2015 to 2018. Participants were patients attending the outpatient clinic of a psychiatric hospital to achieve care for epileptic seizures. Ethical clearance was obtained prior to the conduct of this study. In addition, informed consent was obtained from each patient or their caregivers. Data analysis was done using SPSS version 20. The study involved 177 participants aged 2-70 years (median $=17$ years). The male to female ratio was $2: 1$. The mean duration of illness was $7.8 \pm 5.8$ (range $=1-35$ years). Sensitivity of EEG was $70.6 \%$. There was no difference between either age, gender or duration of illness and probability of having an abnormal EEG. EEG is sensitive in the diagnosis of epilepsy in our setting. Future studies should focus on factors influencing sensitivity of EEG in diagnosis of epilepsy.
\end{abstract}

\section{Introduction}

Epilepsy is one of the most common neurological disorders, affecting about 50 million people worldwide. ${ }^{1}$ According to World Health Organization (WHO), about $80 \%$ of individuals with epilepsy live in Low and Middle Income Countries (LMICs). ${ }^{1}$ It is estimated that about $70 \%$ of People Living With Epilepsy (PLWE) could live seizurefree if their condition is diagnosed and treated early. ${ }^{1}$ Electroencephalography (EEG) is a vital tool in the management of PLWE and other neuropsychiatric disorders. Since its discovery by Hans Berger in 1929, EEG has undergone several modifications to improve its accuracy. ${ }^{2-4}$ Nevertheless, there has been conflicting views on the sensitivity of EEG in diagnosis of epilepsy. While some studies carried out both in the developed countries and in parts of Nigeria reported that the tool has high sensitivity, others observed that it has low sensitivity. ${ }^{5-8}$ Notwithstanding these conflicting views, EEG remains an important complement to history and clinical examination in management of patients with epilepsy. ${ }^{6}$ Therefore it is important to find out the sensitivity of EEG in our setting, since it is widely used in the diagnosis and management of patients with epilepsy.

The main principle of EEG is that it records differences in voltage between two or more points through the use of electrodes placed on the scalp or cortex. ${ }^{9}$ Each disorder that is detected using EEG exhibits characteristic waveforms, which help in its diagnosis. However, it has been noted that some neurologic disorders may exhibit epileptiform waveforms while individual disorders may have different waveform patterns. ${ }^{6}$ In addition, some healthy adults may show epileptiform discharges on EEG. ${ }^{10}$ These phenomena may reduce the accuracy of EEG in the diagnosis of neuropsychiatric disorders. Nevertheless, EEG is essential in diagnosis and classification of epilepsy since there is a tendency to miss or misclassify epilepsy without an EEG. ${ }^{11}$

Studies on sensitivity of EEG reported wide range of values, from $17.3 \%$ to $89 \% .^{12,13}$ In a study of 104 patients by Weenink et al. in The Netherlands, sensitivity of EEG was found to be $63 \%$ (95\% CI: 44-79\%). ${ }^{14}$ However, their study excluded patients younger than 6 years. Just like studies from the developed world, studies from low- and middle-income countries showed wide range of sensitivity for the EEG. In a study by Chowdhury et al. in Bangladesh, sensitivity of EEG was found to be $66 \%(95 \%$ CI $62-68 \%) .{ }^{8}$ In a study of EEG patterns of patients with epilepsy in Kano, Northwestern Nigeria, Owolabi et al. reported that $58.8 \%$ of them had abnormal EEG. ${ }^{15}$ Several factors influence the diagnostic yield of EEG in epilepsy and other neuropsychiatric disorders. According to Chowdhury et al., patient characteristics and differences in the threshold of EEG readers to detect a positive result may influence their interpretation of EEG. ${ }^{8}$ In a meta-analysis of 15 studies, Bouma et al. reported that among adult studies of epilepsy, pooled sensitivity of EEG was $17.3 \%$ (95\% CI: $7.9-33.8 \%$ ) while among child studies the sensitivity was higher at $57.8 \%$ (95\% confidence interval: 49.7$65.6 \%) .{ }^{16}$ However, their study was restricted to patients with first onset epilepsy and did not factor in other patients' characteristics in the analysis. A retrospective study by Watson et al. revealed that EEG has similar sensitivity in detecting
Correspondence: Shehu Sale, Federal Neuropsychiatric Hospital, PMB 2196, Kware, Sokoto State.

Tel.: +2347034631928

E-mail: shehusale@yahoo.com

Key words: EEG, epilepsy, sensitivity, Nigeria.

Conflict of interest: the author declares no potential conflict of interest.

Ethics approval and consent to participate: Ethical clearance was obtained prior to the conduct of this study. Informed consent was obtained from each patient or their caregivers.

Received for publication: 5 March 2020.

Revision received: 12 April 2020.

Accepted for publication: 12 April 2020.

This work is licensed under a Creative Commons Attribution NonCommercial 4.0 License (CC BY-NC 4.0).

(C) Copyright: the Author(s), 2019

Licensee PAGEPress, Italy

Pyramid Journal of Medicine 2020; 3:79

doi:10.4081/pjm.2020.79

seizures in the elderly compared to those of younger age groups. ${ }^{17}$ Another factor affecting the sensitivity of EEG is whether it is done following sleep deprivation or ambulatory. According to Weenink et al., ambulatory EEG was found to be more sensitive than sleep deprived EEG. ${ }^{14}$ Similarly, automatic analysis of EEG signals is more accurate than complete visual analysis. ${ }^{18}$ However, we do not have the equipment for automatic analysis of EEG in our setting. This study therefore aims to determine the sensitivity of EEG in the diagnosis of epilepsy in a neuropsychiatric hospital in northwestern Nigeria.

\section{Materials and Methods}

This was a cross-sectional study conducted from January 2015 to December 2018. Participants were outpatients who were diagnosed with epilepsy in the outpatient clinic of a Neuropsychiatric hospital in Kware, northwestern Nigeria.

\section{Ethical considerations}

Prior to commencement of this study, ethical approval was obtained from the ethical committee of the hospital. In addition, informed consent was obtained from all the participants who had the capacity to give it while assent of parents or caregivers was obtained in other cases. 


\section{Data collection}

Data for this study was obtained by trained research assistants using structured socio-demographic questionnaire and clinical records of the patients. Diagnosis of epilepsy was made by psychiatrists according to criteria set in $10^{\text {th }}$ edition of International Classification of Disease (ICD 10). EEG was done and interpreted by psychiatrists in the hospital after which the research assistants recorded the findings for each patient.

\section{Procedure}

Each consecutive patient diagnosed of epilepsy by psychiatrists in the clinic was approached by the research assistants who informed them about the study. Participants who consented were enrolled into the study and their socio-demographic data were obtained. In addition, the diagnosis was obtained from the clinical records of the patients. After that the participants were directed to the EEG room where the procedure was carried out on them. Subsequently the research assistants obtained the result of the EEG from the psychiatrist and recorded in the patient's form.

\section{Data analysis}

Data obtained from the participants were analyzed using the Statistical Package for Social Sciences version 20. Categorical variables were reported as frequencies and percentages while continuous variables were reported using means and standard deviations. Sensitivity of EEG was determined using the cross-tab function of SPSS. Chi-squared test was carried out to determine differences in sensitivity of EEG across categorical variables. Level of significance was set as $p$ value $<0.05$.

\section{Results}

\section{Socio-demographic characteristics of the study participants}

One hundred and seventy seven patients aged 2-70 years (median= 17 years) participated in this study. Participants aged 11-20 years made up the largest age group in the study, accounting for $41.2 \%$ of the respondents while the least age group was those above 40 years, accounting for $6.2 \%$ of the participants. The male to female ratio was $2: 1$ and only $51(28.8 \%)$ of them had formal education. Majority of the participants, $168(95.0 \%)$ belong to Hausa/Fulani ethnicity (Table 1).

\section{Clinical characteristics of the study participants}

Duration of illness ranged between 1-35 years (mean $=7.8 \pm 5.8)$ while $24(13.6 \%)$ of the patients had family history of epilepsy. All of the patients were on Carbamazepine (Table 1).

\section{EEG Findings of the study partici- pants}

One hundred and twenty-five (70.6\%) of the patients were detected as having epilepsy using EEG (true positive) while 52 (29.4\%) had normal EEG (false negative). Therefore, sensitivity of EEG in diagnosis of epilepsy was $70.6 \%$ (Figure 1). There was no difference in the sensitivity across age, gender and duration of illness with $\mathrm{p}=0.194$, 0.414 and 0.409 respectively (Table 2 ).

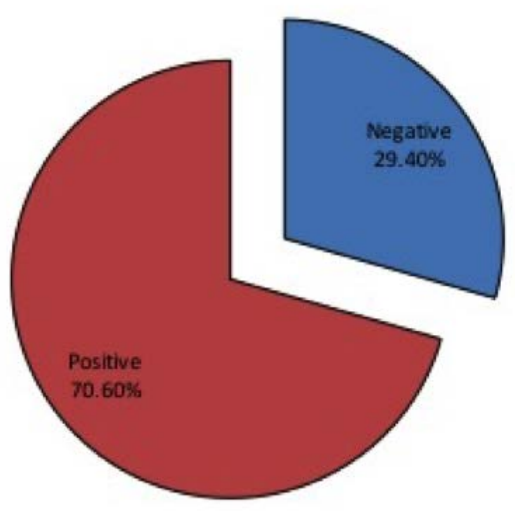

Figure 1. EEG results of the study participants.

Table 1. Socio-demographic and clinical characteristics of the study's participants.

\begin{tabular}{lcc} 
Variable & Frequency & Percentage (\%) \\
Age (years) & & 24.3 \\
$1-10$ & 43 & 41.2 \\
$11-20$ & 73 & 15.3 \\
$21-30$ & 27 & 13.0 \\
$31-40$ & 23 & 6.2 \\
$>40$ & 11 & \\
Median & 17 years & \\
Gender & & 66.7 \\
Male & 118 & 33.3 \\
Female & 59 & 28.8 \\
Formal education (Yes) & 51 & \\
Ethnicity & & 95.0 \\
Hausa/Fulani & 168 & 2.8 \\
Igbo & 5 & 2.2 \\
Others & 4 & 13.6 \\
Family history of epilepsy & 24 & \\
Duration of illness & & 48.6 \\
1-5 years & 86 & 26.0 \\
6-10 years & 46 & 25.4 \\
$>10$ years & 45 & \\
Mean & $7.8 \pm 5.8$ & \\
\hline
\end{tabular}

Table 2. Relationship between some socio-demographic and clinical characteristics of the participants and EEG result.

\begin{tabular}{lccc} 
Variables & \multicolumn{2}{c}{ EEG result } & P \\
& Negative & Positive & 0.414 \\
Gender & & & \\
Male & $37(31.4)$ & $81(68.6)$ & \\
Female & $15(25.4)$ & $44(74.6)$ & 0.194 \\
Age (years) & & & \\
$1-10$ & $9(20.9)$ & $34(79.1)$ & \\
$11-20$ & $25(34.2)$ & $48(65.8)$ & \\
$21-30$ & $5(18.5)$ & $22(81.5)$ & \\
$31-40$ & $10(43.5)$ & $13(56.5)$ & \\
$>40$ & $3(27.3)$ & $8(72.7)$ & \\
\hline Duration of illness & & & \\
$1-5$ & $26(30.2)$ & $60(69.8)$ & \\
$6-10$ & $16(34.8)$ & $30(65.2)$ & \\
$>10$ & $10(22.2)$ & $35(77.8)$ & \\
\hline
\end{tabular}




\section{Discussion}

Epilepsy is a common neuropsychiatric disorder, affecting both the young and the old. ${ }^{1}$ In this study, the median age was 17 years and most of the participants were below 30 years. This is similar to the findings in several studies where majority of the participants were of young age groups. ${ }^{8,19,20}$ Adverse neonatal outcomes like birth asphyxia and brain infections, along with neurological disorders have been shown to predispose children to epileptic seizures. ${ }^{21}$ The male to female ratio was $2: 1$. Several hospital and population-based studies of epilepsy have shown male preponderance..$^{8,19,20}$ In fact, Chowdhury et $a l$. observed that this male preponderance is maintained even after controlling for other factors that significantly predispose more males to epilepsy (for example head injury and stroke). ${ }^{8}$

All of the patients were on Carbamazepine as an anti-epileptic drug. This might be because this was the drug that was readily available in the setting and was more affordable than other anti-epileptics. It has been shown that carbamazepine monotherapy has good tolerability and is highly effective in the treatment of epilepsy. ${ }^{22}$ Family history of epilepsy was only found among $13.6 \%$. This agrees with previous studies that showed only few individuals with epilepsy had family history of epilepsy. ${ }^{23-25}$ However, the genetic predisposition and pattern of inheritance of epilepsy among Nigerians should be explored in future studies.

Sensitivity of EEG was found to be $70.6 \%$. This agrees with several studies that show that EEG is sensitive in detecting individuals with epilepsy. ${ }^{8,14}$ EEG is an important adjunct to clinical information in the diagnosis of epilepsy. Indeed without EEG, diagnosis of epilepsy tends to be missed and the various epileptic classes may be wrongly identified. ${ }^{11}$ However, the level of sensitivity in this study is higher than most of the previous studies. ${ }^{8,14}$ The difference may be due to variations in patient characteristics, study methodology and expertise of the EEG reader. These factors have been shown to influence the diagnostic accuracy of EEG. ${ }^{8}$

It is also interesting that in this study, sensitivity was not different across age groups, gender and regardless of duration of illness. This agrees with a study by Ajiboye et al. in Northcentral, Aina et al. in Southwestern Nigeria and Weenink et al. in The Netherlands. ${ }^{14,19,23}$ The implication of this finding is that EEG can reliably complement clinical data in the diagnosis of epilepsy among all age groups and gender.
This finding differed from a study by Bouma et al. which showed that sensitivity was higher among children compared to elderly patients. ${ }^{12}$ However, their study was restricted to patients with first onset epilepsy. ${ }^{12}$

\section{Limitations}

This study has some limitations. There is no control arm group so it was not possible to get specificity of EEG. In addition, only patients with generalized tonic clonic convulsion could be recruited into the study so it was not possible to determine sensitivity in different epileptic seizure disorders.

\section{Conclusions}

EEG remains an important diagnostic tool in the management of patients with epilepsy. It is sensitive in the diagnosis of epilepsy across all age groups and gender. Notwithstanding its sensitivity, EEG findings must be interpreted in line with clinical data as some patients with epilepsy may show normal EEG patterns. Future studies should focus on factors influencing the sensitivity of EEG in the diagnosis of epilepsy.

\section{References}

1. World Health Organization, Epilepsy. Available from: https://www.who.int/news-room/factsheets/detail/epilepsy. Accessed: Feb 23, 2020.

2. Tudor M, Tudor L, Tudor KI. Hans Berger (1873-1941) — the history of electroencephalography. Acta Med Croatica 2005;59:307-13.

3. Gururangan K, Razavi B, Parvizi J. Diagnostic utility of eight-channel EEG for detecting generalized or hemispheric seizures and rhythmic periodic patterns. Clin Neurophysiol Pract 2018;3:65-73.

4. Suarez-Revelo J, Ochoa-Gomez J, Duque-Grajales J. Improving test-retest reliability of quantitative electroencephalography using different preprocessing approaches. In: Proceedings of the Annual International Conference of the IEEE Engineering in Medicine and Biology Society, EMBS. Institute of Electrical and Electronics Engineers Inc.; 2016:961-4.

5. Paliwal P, Wakerley BR, Yeo LLL, et al. Early electroencephalography in patients with Emergency Room diagnoses of suspected new-onset seizures: Diagnostic yield and impact on clinical decision-making. Seizure 2015;31:22-6.

6. Owolabi L, Owolabi S, Shehu S, Umar M. Interictal electroencephalography in patients with epilepsy in northwestern Nigeria. Ann Niger Med 2013;7:48.

7. Tu B, Young GB, Kokoszka A, et al. Diagnostic accuracy between readers for identifying electrographic seizures in critically ill adults. Epilepsia Open 2017;2:67-75.

8. Chowdhury AH, Chowdhury RN, Khan SU, et al. Sensitivity and Specificity of Electroencephalography (EEG) Among Patients Referred to an Electrophysiology Lab in Bangladesh. J Dhaka Med Coll 2015;23:215-22.

9. MacDonald

Electroencephalography: Basic Principles and Applications. In: International Encyclopedia of the Social \& Behavioral Sciences: Second Edition. Elsevier Inc.; 2015:353-63.

10. Tripathi M, Mehendiratta MM. Role of EEG in Epilepsy. In: Epilepsy Topics. InTech; 2014.

11. Igwe SC, Brigo F, Beida O. Patterns of diagnosis and therapeutic care of epilepsy at a tertiary referral center in Nigeria. Epilepsia 2014;55:442-7.

12. Bouma HK, Labos C, Gore GC, et al. The diagnostic accuracy of routine electroencephalography after a first unprovoked seizure. Eur J Neurol 2016;23:455-63.

13. Chen DK, Graber KD, Anderson CT, Fisher RS. Sensitivity and specificity of video alone versus electroencephalography alone for the diagnosis of partial seizures. Epilepsy Behav 2008;13:115-8.

14. Geut I, Weenink S, Knottnerus ILH, van Putten MJAM. Detecting interictal discharges in first seizure patients: ambulatory EEG or EEG after sleep deprivation? Seizure 2017;51:52-4.

15. Owolabi LF, Sale S, Owolabi SD, et al. Electroencephalography abnormalities in generalized epilepsy and their predictors: A multicenter experience. Ann Afr Med 2018;17:64-9.

16. Bouma HK, Labos C, Gore GC, et al. The diagnostic accuracy of routine electroencephalography after a first unprovoked seizure. Eur J Neurol 2016;23:455-63.

17. Watson P, Conroy A, Moran G, Duncan S. Retrospective study of sensitivity and specificity of EEG in the elderly compared with younger age groups. Epilepsy Behav 2012;25:408-11.

18. Ahammad N, Fathima T, Joseph P. Detection of Epileptic Seizure Event and Onset Using EEG. 2014.

19. Ajiboye PO, Abiodun OA, Ogbebor AI. 
An investigation of the patterns and outcomes of electroencephalographic (EEG) recording requests in the management of neuropsychiatric disorders in a teaching hospital in Nigeria. Afr Health Sci 2017;17:852-8.

20. Olisah V, Adekeye O, Okpataku C, Eseigbe E. Electroencephalographic findings in patients referred for electroencephalogram in a University Teaching Hospital in Northern Nigeria. Sahel Med J 2015;18:78.

21. Ogunlesi T, Ogundeyi M, Olowu A.
Pattern of childhood epilepsies in Sagumu, Nigeria. Indian J Pediatr 2009;76:385-9.

22. Trinka E, Ben-Menachem E, Kowacs PA, et al. Efficacy and safety of eslicarbazepine acetate versus controlled-release carbamazepine monotherapy in newly diagnosed epilepsy: A phase III double-blind, randomized, parallel-group, multicenter study. Epilepsia 201;59:479-91.

23. Aina OF, Ogun OC, Ladapo HTO, et al. Clinical neuropsychiatric correlates and
EEG findings among children with developmental disorders in Lagos, Nigeria. African J Psychiatry (South Africa) 2008;11:123-7.

24. Fields M, Sazgar M, Harden CL. Epilepsy inheritance. In: Controversies in Caring for Women with Epilepsy: Sorting Through the Evidence. Springer International Publishing; 2016:81-6.

25. Bianchi A, Viaggi S, Chiossi E,et al. Family study of epilepsy in first degree relatives: Data from the Italian Episcreen study. Seizure 2003;12:203-10. 